\title{
MicroRNA expression signature and target prediction in familial and sporadic primary macronodular adrenal hyperplasia (PMAH)
}

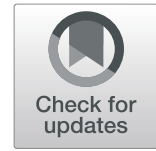

\author{
Xiao-Gang Tan ${ }^{1}$, Jie Zhu ${ }^{2}$ and Liang Cui ${ }^{3^{*}}$
}

\begin{abstract}
Background: Primary macronodular adrenal hyperplasia (PMAH), previously termed ACTH-independent macronodular adrenal hyperplasia (AIMAH), is a rare cause of Cushing's syndrome usually characterized by functioning adrenal macronodules and increased cortisol production.

Methods: To screen and analyse the microRNA (miRNA) profile of PMAH in order to elucidate its possible pathogenesis, a miRNA microarray was used to test tissue samples from patients with familial PMAH, patients with sporadic PMAH and normal control samples of other nontumour adrenocortical tissues and identify characteristic microRNA expression signatures. Randomly selected miRNAs were validated by quantitative real-time reverse transcription polymerase chain reaction (qRT-PCR). Furthermore, the key signalling pathways and miRNAs involved in PMAH pathogenesis were determined by gene ontology and pathway analysis.

Results: Characteristic microRNA expression signatures were identified for patients with familial PMAH (16 differentially expressed microRNAs) and patients with sporadic PMAH (8 differentially expressed microRNAs). The expression of the selected miRNAs was confirmed by qRT-PCR, suggesting the high reliability of the miRNA array analysis results. Pathway analysis showed that the most enriched pathway was the renal cell carcinoma pathway. Overexpression of miR-17, miR-20a and miR-130b may inhibit glucocorticoid-induced apoptosis in PMAH pathogenesis.
\end{abstract}

Conclusion: We identified the miRNA signatures in patients with familial and sporadic PMAH. The differentially expressed miRNAs may be involved in the mechanisms of PMAH pathogenesis. Specific miRNAs, such as miR-17, miR-20a and miR-130b, may be new targets for further functional studies of PMAH.

Keywords: Primary macronodular adrenal hyperplasia (PMAH), microRNA expression, Familial, Sporadic, Pathway analysis, Pathogenesis

\section{Background}

ACTH-independent macronodular adrenal hyperplasia (AIMAH, OMIM \#615954) is also called primary bilateral macronodular adrenal hyperplasia, and the term AIMAH has been recently suggested to be revised to primary macronodular adrenal hyperplasia (PMAH), which

\footnotetext{
* Correspondence: liangcui2018@sina.com

${ }^{3}$ Department of Urology Surgery, Civil Aviation General Hospital, Beijing 100123, China

Full list of author information is available at the end of the article
}

is a rare disorder characterized by bilateral macronodular hyperplasia of the adrenal glands with increased cortisol production. Patients typically present in the fifth and sixth decades of life with obvious clinical manifestations of moon face, sanguineous temperament, skin thinning, buffalo hump and supraclavicular fat pad thickening [1, 2]. PMAH accounts for fewer than $1 \%$ of cases of adrenocorticotropic hormone-independent Cushing's syndrome, and the extent of cortisol excess ranges from subclinical to overt Cushing's syndrome

(c) The Author(s). 2021 Open Access This article is licensed under a Creative Commons Attribution 4.0 International License, which permits use, sharing, adaptation, distribution and reproduction in any medium or format, as long as you give appropriate credit to the original author(s) and the source, provide a link to the Creative Commons licence, and indicate if changes were made. The images or other third party material in this article are included in the article's Creative Commons licence, unless indicated otherwise in a credit line to the material. If material is not included in the article's Creative Commons licence and your intended use is not permitted by statutory regulation or exceeds the permitted use, you will need to obtain permission directly from the copyright holder. To view a copy of this licence, visit http://creativecommons.org/licenses/by/4.0/ The Creative Commons Public Domain Dedication waiver (http://creativecommons.org/publicdomain/zero/1.0/) applies to the data made available in this article, unless otherwise stated in a credit line to the data. 
[3]. The production of cortisol in patients with PMAH is often aberrantly modulated by other hormones, such as vasopressin, gonadotropins, angiotensin, gastric inhibitory peptide and catecholamines, and endocrine disturbance is associated with abnormal adrenocortical sensitivity to various hormonal secretagogues. Furthermore, abnormal activity of a few hormone receptors has been considered to be a causative factor of PMAH [4, 5]. Although the disease phenotype occurs sporadically in the majority of affected patients with PMAH, several reports of familial clustering have been reported [6, 7]. Recently, with the development of gene sequencing technology, gene mutations related to familial and sporadic PMAH, such as ARMC5 mutations found by DNA sequencing [8] and whole-genome sequencing [9] and EDNRA mutations found by whole-exome sequencing [10], have been identified. PMAH presents a bimodal age distribution with a rare subset of patients presenting in the first years of life, particularly with McCuneAlbright syndrome. Most patients with PMAH present in the fifth and sixth decades of life, a later age of onset than that of unilateral adenoma or Cushing's syndrome [2, 11]. Although reports of large families with PMAH have suggested that the phenotype is consistent with a monogenetic, probable autosomal dominant inheritance pattern [12], the genetic or epigenetic basis of familial PMAH remains undetermined.

MicroRNA (miRNA)-associated research has generated great interest since the identification of the first microRNA owing to the extensive potential effects of miRNAs on the regulation of gene expression at both the transcriptional and posttranscriptional levels [13]. MicroRNAs are a class of single-stranded, noncoding small RNAs with a length of approximately 22 nucleotides $[14,15]$. MiRNAs are first transcribed by RNA polymerase II in hairpin structures and are then processed by the RNase III Drosha to become long precursor miRNAs (pre-miRNAs). Then, the RNase III Dicer is involved in the processing from precursor to mature microRNAs, which can bind to the 3' untranslated regions (3'UTRs) of their targeted messenger RNAs in a sequence-specific manner with complete or partial complementarity, leading to degradation or translational repression of the messenger RNA transcript, respectively [16]. The biological functions of only a small fraction of the identified miRNAs have been elucidated, and these miRNAs may be involved in essential regulatory processes such as proliferation, differentiation, apoptosis and development [17]. Although microRNA expression profiles differ among cancer types, it has been demonstrated that several microRNAs might play very specific roles in tumorigenesis by regulating a series of pathways [18-23].

PMAH is rare and generally presents as a sporadic disease. Familial clustering of PMAH is comparatively infrequent. In the past decade, PMAH-affected families have been diagnosed in the General Hospital of the People's Liberation Army. Three out of seventeen members were diagnosed with typical or subclinical PMAH syndrome (Fig. 1). We collected samples from the affected patients from this familial pedigree and adrenal lipoma patients with complete clinical information by adrenalectomy. We previously performed whole-genome sequencing with samples from the three patients with familial PMAH [9]. The samples from the three patients were analysed to identify somatic mutations in ARMC5 exons. We believe that the microRNA expression signature may provide additional information to supplement the whole-genome analysis previously performed in this set of patients. The aims of the study were to identify differentially expressed miRNAs in the samples from patients with familial PMAH, patients with sporadic PMAH and normal control samples from adrenal lipoma patients via a high-throughput approach and to conduct bioinformatics analysis to determine the potential functions of the differentially expressed miRNAs and possible pathways mediating the pathogenesis of PMAH through Gene Ontology (GO) and Kyoto Encyclopedia of Genes and Genomes (KEGG) pathway analyses, respectively.

\section{Methods}

\section{Clinical samples}

All procedures were performed in accordance with the relevant guidelines and regulations. Written informed consent for genetic studies was obtained prior to initiating this study in agreement with protocols approved by the institutional review board at the General Hospital of the People's Liberation Army. This research was reviewed and approved by the ethics committee of General Hospital of the People's Liberation Army. Adrenal nodule samples from patients with familial and sporadic PMAH were collected after surgery. Normal control tissues were collected from the nontumour region of patients with large adrenal lipomas; this region was located $\geq 2 \mathrm{~cm}$ from the lipoma after surgery. Moreover, computed tomography (CT) imaging and haematoxylin and eosin (H\&E) staining were employed to identify the characteristics of nodular hyperplasia. For CT analysis, the images were reviewed by two experienced observers who arrived at a consensus. The imaging characteristics were recorded. For $\mathrm{H} \& \mathrm{E}$ staining, all surgical specimens were fixed with $10 \%$ buffered formaldehyde and routinely processed for histologic diagnosis. The tumour histology was independently confirmed by 2 pathologists (Fig. 2). On the basis of the pathological diagnosis 


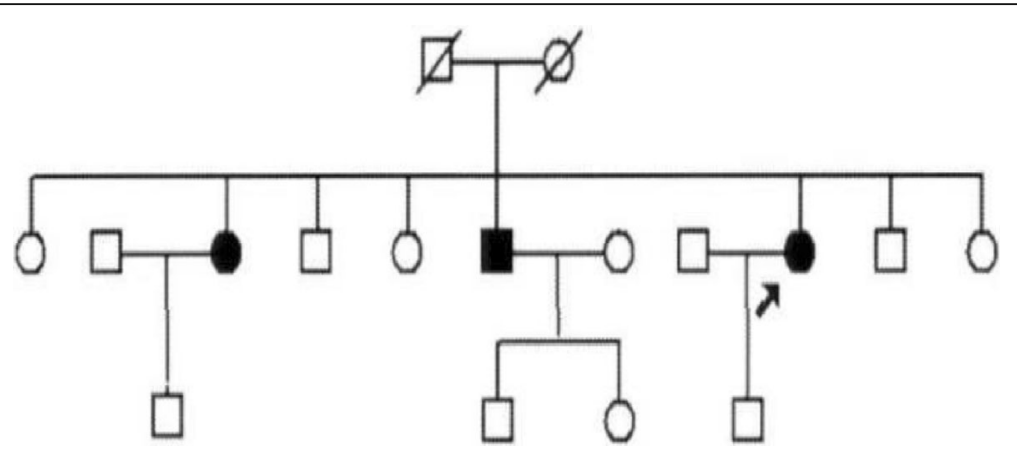

Fig. 1 Pedigree of the familial patients with PMAH. Arrow: proband

correction, 3 to 5 tissue pieces of approximately $3 \mathrm{~mm}^{3}$ according to the size of the lesion were cut and put into a $1 \mathrm{ml}$ Eppendorf (EP) tube. The samples were snap frozen in liquid nitrogen immediately after resection and stored at $-80^{\circ} \mathrm{C}$ until RNA extraction. We used one sample for whole-genome sequencing and another sample for microRNA profile screening.

\section{Total RNA extraction}

Total RNA from the adrenal gland tissue removed from patients with familial or sporadic PMAH as well as normal control tissue was extracted according to the standard protocol.

\section{MicroRNA array analysis}

An Affymetrix GeneChip miRNA Array (Affymetrix, Santa Clara, CA) was applied to investigate the differential microRNA expression profile in familial and sporadic PMAH samples compared with normal control samples. This microRNA array contained 46,228 probes, which composes 7815 probe sets, including controls, and covers 71 organisms, including humans, mice, rats, canines and rhesus macaques. The array content was derived from the Sanger miRBase miRNA database v.11 (April 15, 2008). In brief, $1.5 \mu \mathrm{g}$ of total RNA extracted from different groups of samples was labelled using a 3DNA Array Detection FlashTag ${ }^{\text {tix }}$ RNA Labeling Kit according to the recommendations of the manufacturer.

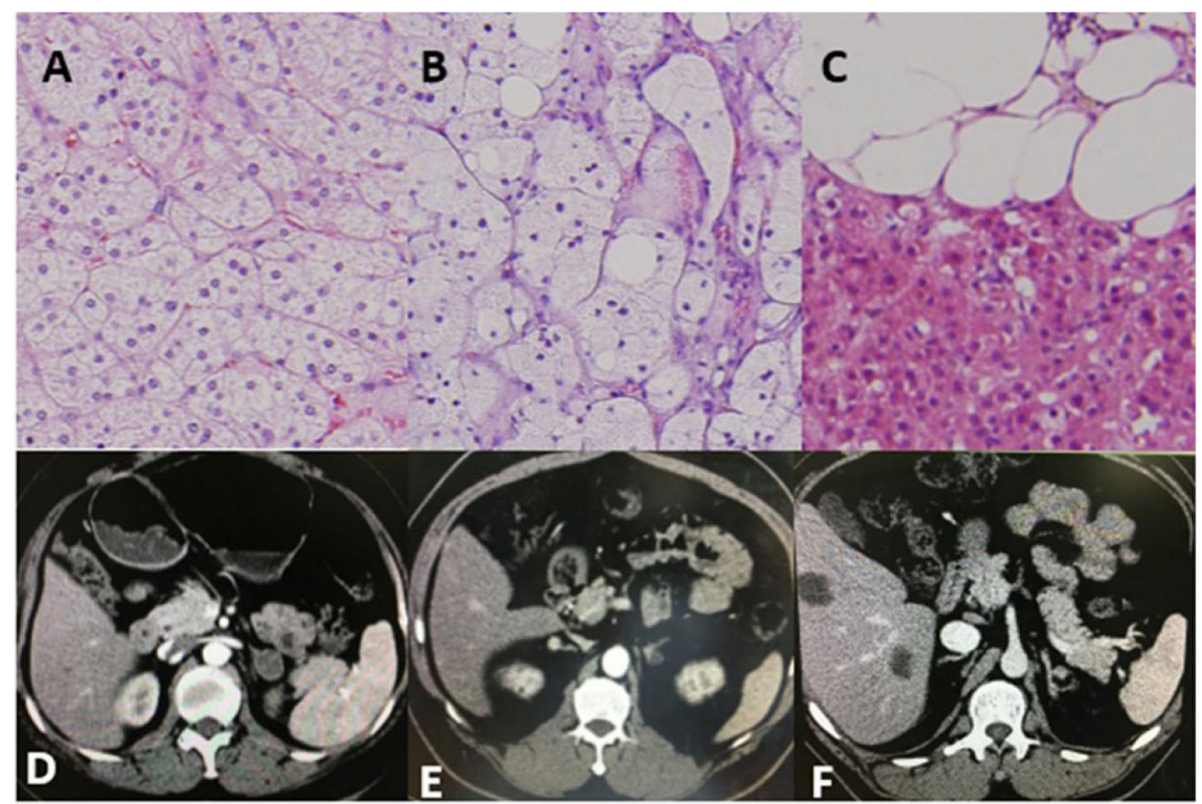

Fig. 2 H\&E staining (200X) and CT imaging. A Left adrenal nodule of familial PMAH H\&E staining imaging. B Left adrenal nodule of sporadic PMAH H\&E staining imaging. C Left adrenal lipoma of the normal control H\&E staining imaging. $\mathbf{D}$ Left adrenal nodule of familial PMAH CT imaging. E Left adrenal nodule of sporadic PMAH CT imaging. $\mathbf{F}$ Left adrenal lipoma of the normal control CT imaging 
First, the addition of a poly(A) tail was carried out at $37^{\circ} \mathrm{C}$ for $15 \mathrm{~min}$ in a $15 \mu \mathrm{l}$ reaction mixture, which contained $1 \times$ Reaction Buffer, $1.5 \mathrm{ml}$ of $\mathrm{MgCl}_{2}, 1 \mu \mathrm{l}$ of ATP Mix diluted 1:500 and $1 \mu \mathrm{l}$ of PAP enzyme. Second, flash tag ligation was performed at room temperature for 30 min by adding $4 \mu \mathrm{l}$ of $5 \times$ Flash Tag Ligation Mix Biotin and $2 \mu \mathrm{l}$ of T4 DNA ligase into the $15 \mu \mathrm{l}$ reaction mixture. To stop the reaction, $2.5 \mu \mathrm{l}$ of Stop Solution was added. All samples were hybridized, washed and scanned with an Affymetrix Scanner.

\section{MiRNA qRT-PCR}

Reverse transcription (RT) was performed on miRNAs using a TaqMan MicroRNA Reverse Transcription Kit (Applied Biosystems) with incubation at $16^{\circ} \mathrm{C}$ for 30 $\min , 42^{\circ} \mathrm{C}$ for $30 \mathrm{~min}$, and $85^{\circ} \mathrm{C}$ for $5 \mathrm{~min}$ for denaturation of the enzyme. RT was performed at $37^{\circ} \mathrm{C}$ for $1 \mathrm{~h}$ followed by $5 \mathrm{~min}$ at $95^{\circ} \mathrm{C}$. TaqMan microRNA assays (Applied Biosystems) were performed using a 7500 Fast Real-Time PCR System in the "9600 emulation" run mode. Ct values were converted into copy numbers (copy no. $=2^{\wedge}(-\mathrm{Ct})$ ) and normalized to RNU48.

\section{Bioinformatics analysis}

The GO Project provides a controlled vocabulary to describe genes and gene products in any organism (http:// www.geneontology.org). The ontology covers three categories: biological process, cellular component and molecular function. Fisher's exact test was used to determine if there was more overlap between the differentially expressed gene list and the GO annotation list than would be expected by chance. The $P$-value denotes the significance of the enrichment of a GO term with the differentially expressed genes. The lower the $P$-value, the more significant is the GO term (a P-value $<0.05$ is the recommended cut-off criterion).

Pathway analysis is a functional analysis that maps genes to KEGG pathways. The $P$-value (EASE score, Fisher P-value or hypergeometric P-value) denotes the significance of a pathway correlated to the conditions. The lower the P-value, the more significant is the pathway (the recommended cut-off P-value is 0.05).

\section{Statistical analysis}

The results are expressed as the mean \pm SD values. Data for haemodynamic parameters were compared using paired t-tests. Values of $P<0.05$ were considered statistically significant $(\mathrm{P}<0.05$ for differentially expressed miRNAs).

For comparing the differentially expressed miRNA profiles between two groups, fold change values and $P$ values were calculated and used to identify significantly differentially expressed miRNAs (based on the all- isoform value). Differentially expressed miRNAs between two samples were defined by filtering with a fold change criterion (based on the all-isoform value), and hierarchical clustering was then performed. MiRNA targets were predicted by GO analysis, and KEGG pathway analysis was performed based on the differentially expressed miRNAs.

\section{Results}

Expression profiles of microRNAs in patients with familial or sporadic PMAH compared with normal controls

A microRNA microarray analysis was performed on tissue samples from 3 patients with familial PMAH (Group 1 ), tissue samples from 2 patients with sporadic PMAH (Group 2) and 3 normal control tissues (Group 3) to compare the differences in microRNA expression levels. The differences in microRNA expression were analysed between the patients with familial PMAH and the normal controls. A total of 16 microRNAs met the false discovery rate (FDR) cut-off criterion of 0.05 . Seven miRNAs were upregulated (hsa-miR-4306, hsa-miR130a, hsa-miR-20b, hsa-miR-20a, hsa-miR-15a, hsamiR-106a, and hsa-miR-17), and 3 miRNAs were downregulated (hsa-miR-197, hsa-miR-3656, and hsamiR-3196) (Table 1). However, the following microRNAs exhibited statistically significant differential expression, albeit with very low signal intensities: hsamiR-17*, hsa-miR-18b, hsa-miR-1976, hsa-miR-454, hsa-miR-629\%, and hsa-miR-629. After filtering out the low-intensity miRNAs, the raw signal intensities

Table 1 MiRNAs with significantly different expression $(P<0.05)$ between familial patients with PMAH (Group1) and normal control tissues (Group3)

\begin{tabular}{llll}
\hline miRNAs & Fold change & $\boldsymbol{P}$ value & Case/control \\
\hline hsa-miR-4306 & 1.43 & 0.003223 & Up \\
hsa-miR-130a & 3.05 & 0.03120 & Up \\
hsa-miR-20b & 3.11 & 0.003463 & Up \\
hsa-miR-20a & 2.07 & 0.005487 & Up \\
hsa-miR-15a & 1.97 & 0.04733 & Up \\
hsa-miR-106a & 1.98 & 0.01258 & Up \\
hsa-miR-17 & 1.98 & 0.01607 & Up \\
hsa-miR-197 & 0.52 & 0.02572 & Down \\
hsa-miR-3656 & 0.53 & 0.04458 & Down \\
hsa-miR-3196 & 0.51 & 0.006570 & Down \\
hsa-miR-17* & & 0.01237 & Up \\
hsa-miR-18b & & 0.02489 & Up \\
hsa-miR-629 & & 0.03823 & Up \\
hsa-miR-1976 & & 0.03086 & Down \\
hsa-miR-629* & & 0.03518 & Down \\
hsa-miR-454 & & 0.03496 & Down \\
\hline
\end{tabular}



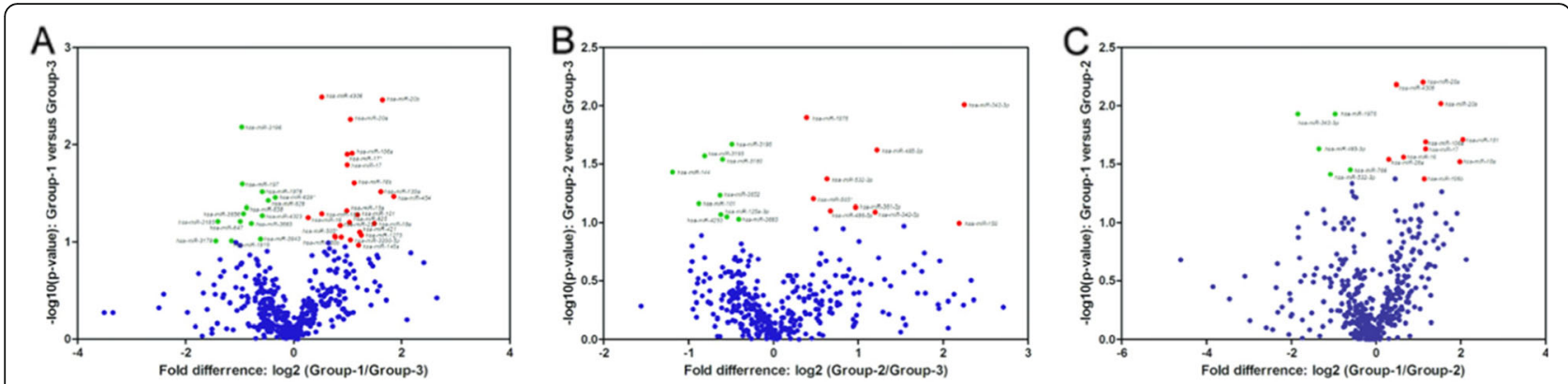

Fig. 3 Volcano plot demonstrating differences in expression levels of microRNAs in 2 groups of PMAH patients and normal controls based on the microarray study. The log-fold changes were plotted based on the log odds of differential expression. The microRNAs with significant differences in expression levels $(p<0.05)$ between familial patients with PMAH and normal controls after Benjamini-Hochberg correction are indicated in $(\mathbf{A})$. The microRNAs with significant differences in expression levels $(p<0.05)$ between sporadic patients with PMAH and normal controls after correction are indicated in (B). The microRNAs with significant differences in expression levels $(p<0.05)$ between familial and sporadic patients with PMAH after correction are indicated in (C)

were normalized to the median value. The differentially expressed miRNAs passed volcano plot filtering (Fig. 3A).

A similar analysis was performed to assess differences in microRNA expression levels between 2 patients with sporadic PMAH and 3 normal controls. Through filtering according to a FDR cut-off criterion of 0.05 and microRNA expression signal intensities, 8 microRNAs were found to be differentially expressed between the above 2 groups; 1 miRNA was upregulated (hsa-miR3196) and 2 miRNAs were downregulated (hsa-miR342-3p and hsa-miR-532-3p) (Table 2). After filtering out the low-intensity miRNAs, the raw signal intensities were normalized to the median value. The differentially expressed miRNAs passed volcano plot filtering (Fig. 3B). The other microRNAs showed no differences in expression between patients with sporadic PMAH and normal controls.

To identify the microRNAs that were differentially expressed between patients with familial and sporadic $\mathrm{PMAH}$, we compared the microRNA expression

Table 2 MiRNAs with significantly different expression $(P<0.05)$ between sporadic cases (Group2) and normal control tissues (Group3)

\begin{tabular}{lcll}
\hline miRNAs & Fold change & P value & Case/control \\
\hline hsa-miR-3196 & 1.41 & 0.02156 & Up \\
hsa-miR-342-3p & 0.21 & 0.009685 & Down \\
hsa-miR-532-3p & 0.65 & 0.04271 & Down \\
hsa-miR-3195 & & 0.0272 & Up \\
hsa-miR-3180 & & 0.0288 & Up \\
hsa-miR-445 & & 0.0370 & Up \\
hsa-miR-1976 & & 0.0125 & Down \\
hsa-miR-485-3p & & 0.0241 & Down \\
\hline
\end{tabular}

profiles of these two independent groups of samples. The same criteria for the FDR cut-off value and expression intensity were applied to filter the candidate microRNAs. Two miRNAs were upregulated (hsamiR-342-3p and hsa-miR-532-3p), and 9 miRNAs were downregulated (hsa-miR-4306, hsa-miR-20b, hsamiR-20a, hsa-miR-106a, hsa-miR-17, hsa-miR-101, hsa-miR-16, hsa-miR-26a, and hsa-miR-106b) (Table 3). After filtering out the low-intensity miRNAs, the raw signal intensities were normalized to the median value. The differentially expressed miRNAs passed volcano plot filtering (Fig. 3C).

Validation of microRNA array analysis results by qRT-PCR To validate the microRNA microarray analysis results, qRT-PCR was performed on total RNA extracted from surgical tissues obtained from patients with familial and

Table 3 MiRNAs with significantly different expression $(P<0.05)$ between familial patients with PMAH (Group1) and sporadic cases (Group2)

\begin{tabular}{llll}
\hline miRNAs & Fold change & P value & Case/control \\
\hline hsa-miR-342-3p & 3.6 & 0.01188 & Up \\
hsa-miR-532-3p & 2.1 & 0.03911 & Up \\
hsa-miR-4306 & 0.71 & 0.006598 & Down \\
hsa-miR-20b & 0.35 & 0.009508 & Down \\
hsa-miR-20a & 0.46 & 0.006258 & Down \\
hsa-miR-106a & 0.44 & 0.04284 & Down \\
hsa-miR-17 & 0.44 & 0.02335 & Down \\
hsa-miR-101 & 0.24 & 0.01947 & Down \\
hsa-miR-16 & 0.64 & 0.02747 & Down \\
hsa-miR-26a & 0.81 & 0.02877 & Down \\
hsa-miR-106b & 0.46 & 0.02046 & Down \\
\hline
\end{tabular}


sporadic PMAH and surgical tissues obtained from normal controls. The expression level of hsa-miR-20b in patients with familial PMAH was 1.56-fold higher than that in normal controls, with a $P$-value of 0.01165 , consistent with the microRNA array analysis results (3.11fold higher, $\mathrm{P}$-value $=0.003463)$. The expression level of hsa-miR-342-3p (downregulated 0.68-fold with Pvalue $=0.005814$ ) was consistent with the result from the microRNA array analysis (hsa-miR-342-3p was downregulated 0.21-fold in patients with sporadic PMAH compared with normal controls). Hsa-miR-342-3p was upregulated 1.58-fold but hsa-miR-101 was downregulated 0.40-fold in patients with familial PMAH compared to patients with sporadic PMAH, consistent with the microRNA array analysis results, in which hsa-miR-342$3 \mathrm{p}$ was upregulated 3.6-fold and hsa-miR-101 was downregulated 0.24-fold in patients with familial PMAH compared with those with sporadic PMAH.

\section{Hierarchical clustering of microRNAs with differential expression}

To identify the unique microRNA expression signatures among different groups of patients, hierarchical clustering analysis was performed on all microRNAs based on the expression signal intensities obtained by microarray analysis. This hierarchical clustering analysis clustered microRNAs together according to their expression levels and clustered samples from different groups based on the similarity of the expression profiles for the investigated microRNAs [24]. By supervised hierarchical clustering with pairwise comparisons among these 3 groups of samples, we could always identify a unique group of microRNAs that were able to differentiate one group from the other. The 16 differentially expressed microRNAs (10 downregulated and 6 upregulated microRNAs) in the signature identified by microarray analysis clearly discriminated between patients with familial PMAH and normal controls (Fig. 4A). Similarly, the microRNA signature containing 8 differentially expressed microRNAs were able to discriminate between patients with sporadic PMAH and normal controls with a statistically significant $P$-value of 0.05 (Fig. 4B). The 11 identified differentially expressed microRNAs were able to discriminate between patients with familial PMAH and patients with sporadic PMAH (Table 3).

\section{Comprehensive target-network prediction and pathway analysis of differentially expressed microRNAs}

Functional analyses of the differentially expressed microRNAs between the PMAH patients and normal controls revealed that some enriched pathways, such as signal transduction, signalling molecules and metabolic interactions, were potentially associated with the pathogenesis of PMAH. For comparative analysis between
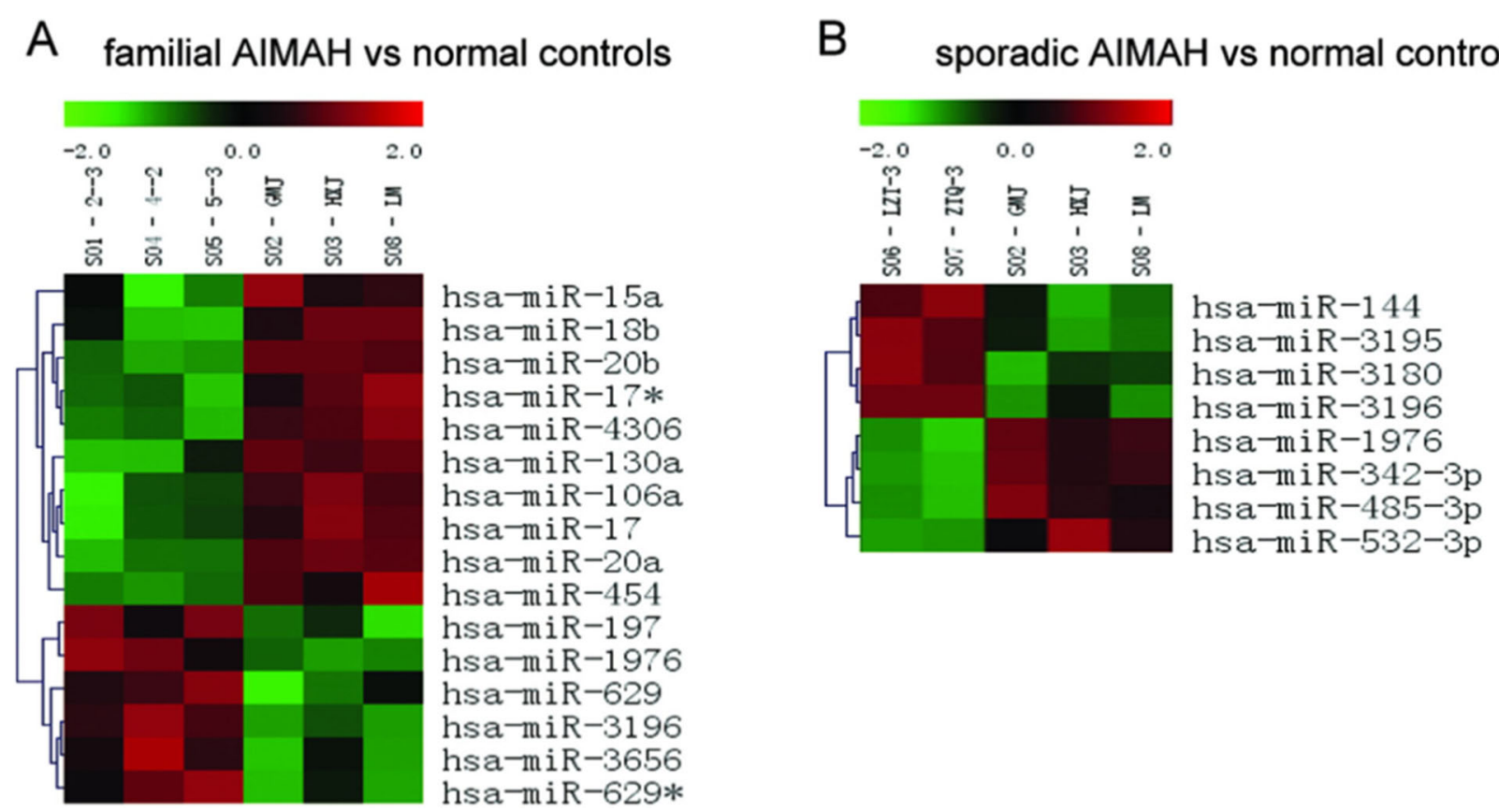

Fig. 4 microRNAs expression signature consisting of diffrentially expressed microRNAs between familial or sporadic PMAH and normal controls. A. Differentially expressed microRNAs $(p<0.05)$ were clustered and the results showed that the expression profiles of these 16 microRNAs could be used to separate between the familial PMAH and normal controls. B. Differentially expressed microRNAs ( $p<0.05)$ were clustered and the results showed that the expression profiles of these 8 microRNAs could be used to separate between the sporadic PMAH and normal controls 
patients with familial PMAH and normal controls, all 16 differentially expressed microRNAs were imported into KEGG pathway analysis software, and the circadian rhythm pathway was the pathway most affected by these microRNAs, containing 6 genes predicted to be potential targets (NPAS2, CRY2, BHLHE40, BHLHE41, CRY1 and CLOCK). However, the renal cell carcinoma pathway, mTOR signalling pathway, glioma pathway, pancreatic cancer pathway and endocytosis pathway were the top signalling pathways affected by these differentially expressed microRNAs (Fig. 5A). When KEGG pathway analysis was similarly performed on the 8 differentially expressed microRNAs between the patients with sporadic PMAH and normal controls, the renal cell carcinoma, dilated cardiomyopathy, axon guidance, ubiquitin-mediated proteolysis, endocytosis and MAPK signalling pathways were ranked as the most affected pathways on the basis of the predicted targets, while the renal cell carcinoma pathway contained 5 genes (CDC42, CUL2, EP300, GRB2 and SLC2A1) that were predicted to be potential downstream targets of the differentially expressed microRNAs (Fig. 5B).

Interestingly, the renal cell carcinoma pathway was one of the top 2 most affected pathways among all associated pathways containing potential targets of the microRNAs that were differentially expressed in patients with either familial or sporadic PMAH compared to normal controls. In the comparison of differentially expressed microRNAs between patients with familial PMAH and normal controls, the renal cell carcinoma pathway contained 25 genes (EGLN3, EGLN2, EGLN1, PAK6, PAK7, CUL2, CDC42, RAC1, SOS2, GAB1,
SLC2A1, TGFA, PAK1, PIK3R1, AKT3, PIK3R2, MAP 2 K1, MET, RAF1, MAPK1, HIF1A, CRKL, VEGFA, RAP1A and CRK) that were targeted by these microRNAs, while 5 genes (CDC42, CUL2, EP300, GRB2 and SLC2A1) were targeted by microRNAs that were differentially expressed between patients with sporadic PMAH and normal controls (Fig. 6).

\section{Discussion}

Recent studies have indicated that microRNA expression signatures might be useful for the characterization and prediction of certain diseases, especially benign or malignant tumours $[25,26]$, but the investigation of microRNA expression signatures in patients with familial PMAH and sporadic PMAH, which have very low incidence rates, remains unclear. In this study, microRNA microarray analysis was applied to identify the differentially expressed microRNAs between patients with familial or sporadic PMAH and normal controls. Our results revealed that a series of differentially expressed microRNAs found by comparisons of different groups could contribute to the characteristics of PMAH.

Before the experiments, the individual performing quality control on the miRNA microarray analysis results randomly selected hsa-miR-20b, hsa-miR-342-3p and hsa-miR-101 for qRT-PCR to validate the microRNA microarray analysis results. We evaluated the expression level of hsa-miR-20b, which, based on our microarray data, was differentially expressed in tissues from patients with familial PMAH compared with normal controls. The expression level of hsa-miR-20b in patients with familial PMAH was 1.56-fold higher than

B sporadic AIMAH versus normal controls

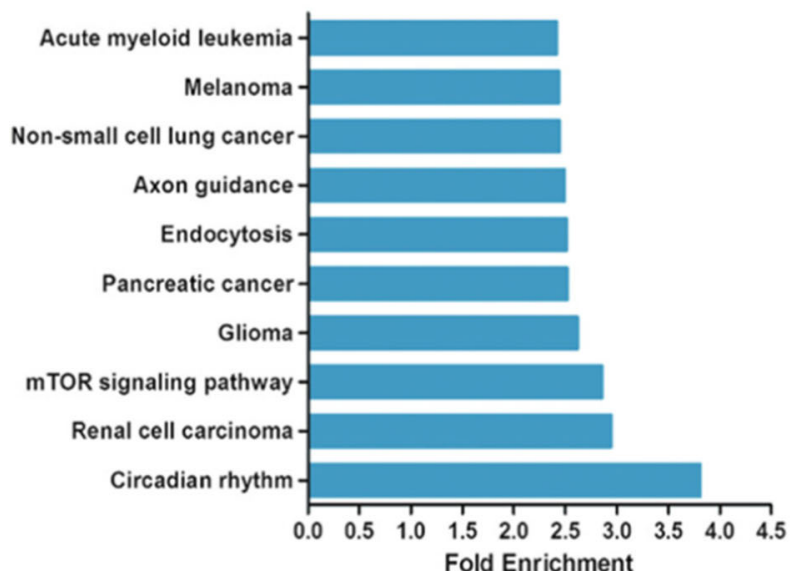

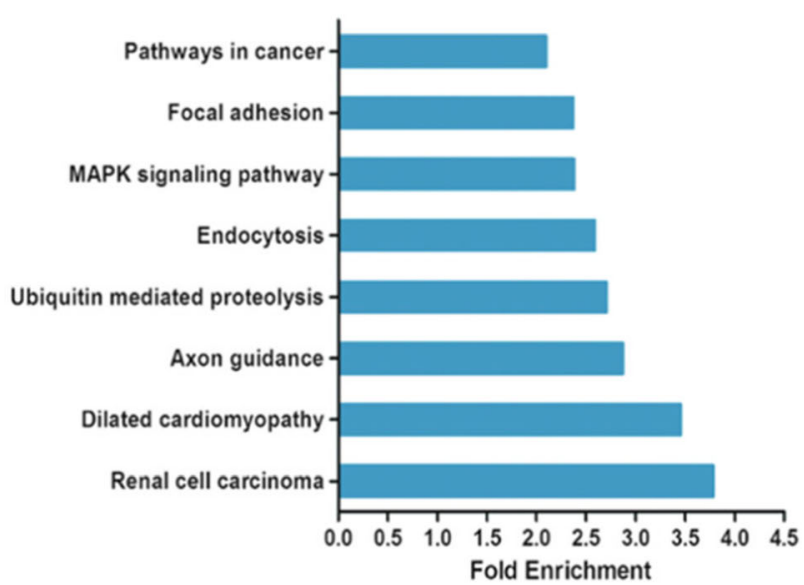

Fig. 5 KEGG pathway analysis on diffrentially expressed microRNAs between familial or sporadic PMAH and normal controls. A. KEGG Pathway analysis was performed on 16 altered microRNAs to reveal that Circadian Rhythm pathway was the most affected pathway. B. KEGG Pathway analysis was performed on 8 altered microRNAs to reveal that renal cell carcinoma pathway was ranked the most affected pathway 


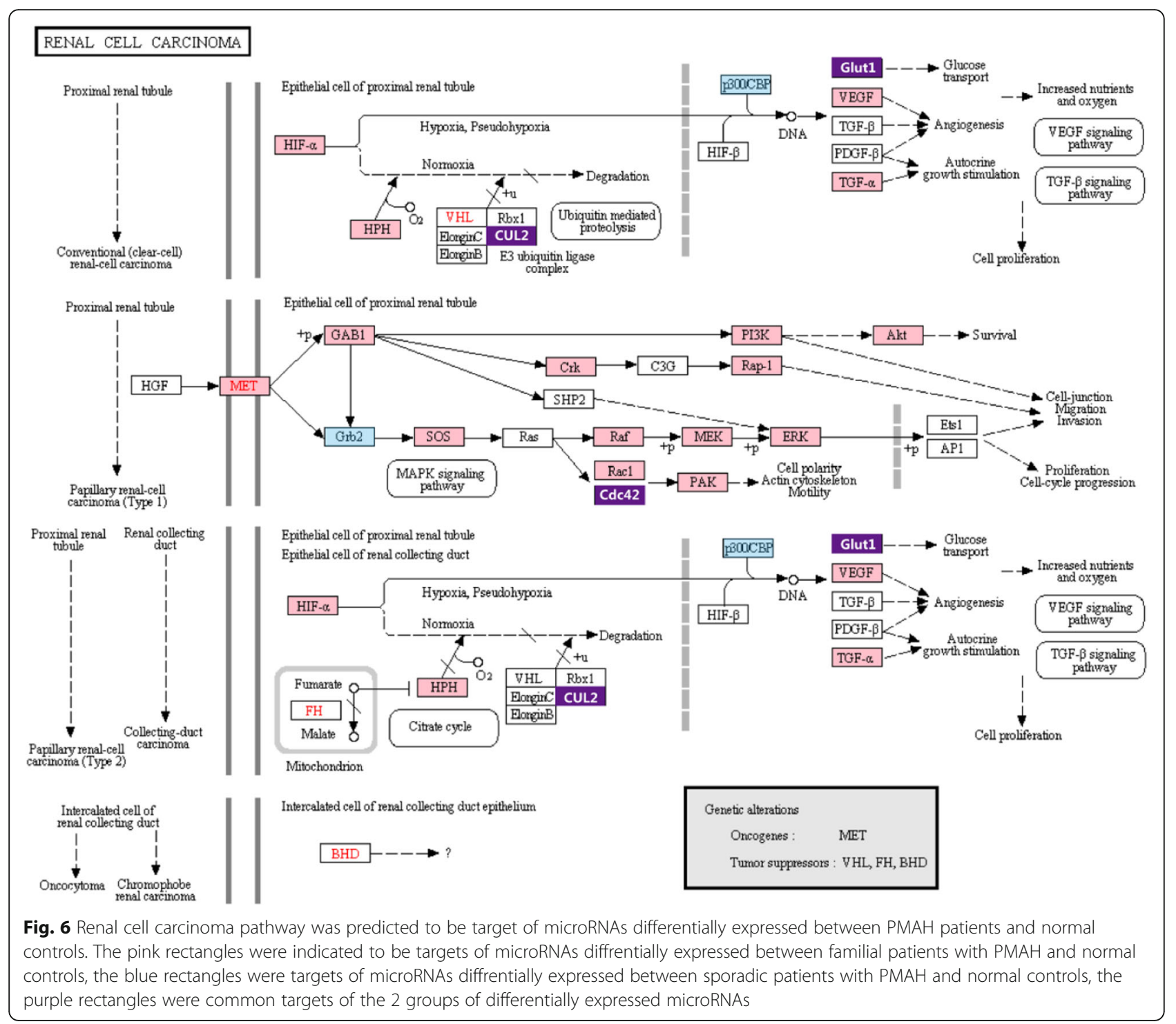

that in normal controls, consistent with the microRNA array analysis results. The expression level of hsa-miR$342-3 p$ was determined by qRT-PCR in samples from patients with sporadic PMAH and normal controls to validate the comparison of the microRNA expression profile between these 2 groups, and the results were consistent with those of the microRNA array analysis. Similarly, hsa-miR-342-3p and hsa-miR-101 were selected to validate the differential microRNA expression levels between patients with familial PMAH and patients with sporadic PMAH. The qRT-PCR results showed that hsa-miR-342-3p was upregulated 1.58-fold but hsa-miR101 was downregulated 0.40 -fold in patients with familial PMAH compared to patients with sporadic PMAH. These qRT-PCR results were consistent with those of the microRNA array analysis, in which hsa-miR-342-3p was upregulated 3.6-fold and hsa-miR-101 was downregulated 0.24-fold in patients with familial PMAH compared with those with sporadic PMAH. Hsa-miR-101, hsa-miR-20b and hsa-miR-342-3p were validated by qRT-PCR, and the results were directly correlated with our microRNA microarray data, suggesting the high reliability of the miRNA array analysis results.

KEGG pathway analysis of the selected microRNAs and further validation by qRT-PCR revealed that these microRNAs had putative downstream targets involved in many important pathways, including the circadian rhythm pathway, renal cell carcinoma pathway, MAPK signalling pathway and other signalling pathways.

In our study, the renal cell carcinoma pathway was ranked as the most affected signalling pathway in both patients with familial PMAH and patients with sporadic 
PMAH compared to normal controls, although PMAH is considered to be a cytologically benign disease without a propensity for invasion and metastasis. In addition, CUL2, CDC42, GLUT1 and other proteins, which have been demonstrated to be critical in malignant renal cell carcinoma, were predicted to be downstream target proteins of microRNAs differentially expressed in PMAH patients compared with normal controls. Our findings in this study were consistent with previous studies from other groups; for instance, the microRNA expression profile for massive macronodular adrenocortical disease shared differentially expressed microRNAs with that for renal cell carcinoma, and these microRNAs were previously implicated in tumorigenesis or metastasis [27]. In another study, several aberrantly expressed genes that were demonstrated to be involved in oncogenic pathways were also identified in one PMAH patient by gene expression profiling [28]. Although PMAH is considered benign because metastasis and invasion have never been reported in long-term postoperative surveillance of patients [29], a massive increase in the adrenocortical cell mass is a typical characteristic of PMAH. The abovementioned results indicate an abnormality in the control of proliferation in adrenocortical cells, which is similar to the characteristics of malignant tumors to some extent. The potential mechanisms underlying abnormalities cell apoptosis might at least partially include the disruption of normal cell cycle progression through alterations in microRNA expression.

MiR-17 and miR-20a, which were significantly upregulated in patients with familial PMAH compared with normal controls, are two of the six miRNAs in the miR17-92 cluster (which contains miR-17, -18a, -19a, -19b$1,-20 \mathrm{a}$, and $-92 \mathrm{a}-1)$ generated from a single precursor RNA that is transcribed from chromosome 13 [30]. Both miR-17 and miR-20a have been reported to contribute to glucocorticoid-induced apoptosis of chondrocytes in lymphoma [31-33]. MiR-17 has been reported to be involved via the miR-17 pathway in glucocorticoidinduced cell death in paediatric acute lymphoblastic leukaemia [34-36]. The NR3C1-targeting miR-130b was found to exhibit higher expression in a glucocorticoidinsensitive multiple myeloma cell line, and its introduction into a glucocorticoid-sensitive line impaired the cellular response to glucocorticoid treatment, including the induction of apoptosis [37]. Therefore, we might predict that overexpression of miR-17, miR-20a and miR-130b can inhibit glucocorticoid-induced apoptosis in PMAH pathogenesis.

Hsa-miR-342-3p, hsa-miR-20b and hsa-miR-101, targeting CDC42, ERK and MEK, respectively, in the MAPK signalling pathway or abnormal expression of PI3K/AKT mediated by differentially expressed microRNAs might interfere with normal apoptosis progression, in turn promoting the survival of adrenocortical cells. In addition, the TGF- $\beta$ signalling pathway and VEGF signalling pathway are involved in the pathogenesis of PMAH.

In the comparison between familial PMAH patients and normal controls, the circadian rhythm pathway was the most affected signalling pathway based on the downstream targets of the differentially expressed microRNAs. Circadian rhythms are universal 24-h oscillation patterns in the metabolic, physiological, endocrinal and behavioural functions of almost all species, and core circadian genes such as PER, CRY and CLOCK seem to be important for tissue homeostasis and tumorigenesis [3840]. In endocrine diseases, key components of the circadian rhythm pathway can interact with steroid hormone receptors to influence the pathogenesis of some endocrine tumours. Core circadian rhythm proteins such as PER2 and CLOCK can be coexpressed with steroid hormone receptors, and CLOCK-associated genes can regulate glucocorticoid activity in almost all tissues by enhancing the transcriptional activity of glucocorticoid receptors (GRs) [41, 42]. One potential underlying mechanism involves acetylation of several lysine residues on GRs and concomitant attenuation of GR binding to glucocorticoid response elements [43]. Because PMAH is characterized by bilateral massive enlargement with dysfunction of glucocorticoid hormones as well as their response receptors, circadian rhythm pathways might play an important role during the development of this disease through their interactions with glucocorticoid receptors.

\section{Conclusion}

Analysis of microRNA expression signatures revealed that the renal cell carcinoma pathway might play an important role in the pathogenesis of PMAH, although $\mathrm{PMAH}$ is a benign hereditary endocrine abnormality without malignant characteristics. Specific microRNAs, such as miR-17, miR-20a and miR-130b, play a role in the pathogenesis and progression of $\mathrm{PMAH}$, and our findings contribute not only to an improved understanding of this benign disease but also to the development of new therapeutic and preventative strategies for PMAH.

\section{Abbreviations \\ PMAH: Primary macronodular adrenal hyperplasia; miRNA: Microrna; qRT- \\ PCR: Quantitative real-time reverse transcription polymerase chain reaction; \\ GO: Gene Ontology; KEGG: Kyoto Encyclopedia of Genes and Genomes; \\ $C T$ : The computed tomograph; H\&E staining: Hematoxylin-eosin staining}

\section{Acknowledgements}

The authors thank Xingyi Hang for his excellent technical assistance.

\section{Authors' contributions}

LC conceived the study, and developed its design; contributed in patients' assessment, sampling and biochemical testing. Also, participated in the analysis of molecular studies and patients' counseling and manuscript 
writing. XG T performed molecular testing, data collection and participated in results analysis and manuscript writing. JZ was involved in the study design, methods validation, data analysis, manuscript writing and review. All authors read and approved the final manuscript.

\section{Funding}

Not applicable.

\section{Availability of data and materials}

The data that support the findings of this study are available from the corresponding author upon reasonable request.

\section{Declarations}

\section{Ethics approval and consent to participate}

This research was reviewed and approved by the ethics committee of General Hospital of the People's Liberation Army. Written informed consent for genetic studies was obtained prior to initiating this study in agreement with protocols approved by the institutional review board at the General Hospital of the People's Liberation Army.

\section{Consent for publication}

Not applicable.

\section{Competing interests}

The authors declare that they have no competing interests.

\section{Author details}

${ }^{1}$ Department of Thoracic Surgery, Xuan Wu Hospital of Capital Medical University, Beijing 100053, China. ${ }^{2}$ Department of Urology Surgery, Chinese PLA General Hospital, Beijing 100082, China. ${ }^{3}$ Department of Urology Surgery, Civil Aviation General Hospital, Beijing 100123, China.

Received: 20 June 2020 Accepted: 5 December 2021

Published online: 05 January 2022

\section{References}

1. Louiset E, Duparc C, Young J, Renouf S, Tetsi Nomigni M, Boutelet I, et al. Intraadrenal corticotropin in bilateral macronodular adrenal hyperplasia. N Engl J Med. 2013;369(22):2115-25. https://doi.org/10.1056/NEJMoa1215245.

2. Verma A, Mohan S, Gupta A. ACTH-independent macronodular adrenal hyperplasia: imaging findings of a rare condition : a case report. Abdom Imaging. 2008:33(2):225-9. https://doi.org/10.1007/s00261-007-9236-y.

3. Lee S, Hwang R, Lee J, Rhee Y, Kim DJ, Chung UI, et al. Ectopic expression of vasopressin $\mathrm{V} 1 \mathrm{~b}$ and $\mathrm{V} 2$ receptors in the adrenal glands of familial ACTHindependent macronodular adrenal hyperplasia. Clin Endocrinol (Oxf). 2005; 63(6):625-30. https://doi.org/10.1111/j.1365-2265.2005.02387.x.

4. Vezzosi D, Cartier D, Régnier C, et al. Familial adrenocorticotropinindependent macronodular adrenal hyperplasia with aberrant serotonin and vasopressin adrenal receptors. Eur J Endocrinol. 2007;156(1):21-31. https:// doi.org/10.1530/eje.1.02324.

5. Nies C, Bartsch DK, Ehlenz K, Wild A, Langer P, Fleischhacker S, et al. Familial ACTH-independent Cushing's syndrome with bilateral macronodular adrenal hyperplasia clinically affecting only female family members. Exp Clin Endocrinol Diabetes. 2002;110(6):277-83. https://doi.org/10.1055/s-2002-34 590.

6. Miyamura N, Taguchi T, Murata Y, Taketa K, Iwashita S, Matsumoto K, et al. Inherited adrenocorticotropin-independent macronodular adrenal hyperplasia with abnormal cortisol secretion by vasopressin and catecholamines: detection of the aberrant hormone receptors on adrenal gland. Endocrine. 2002;19(3):319-26. https://doi.org/10.1385/ENDO:19:3:319.

7. Vezzosi D, Bennet A, Rochaix P, Courbon F, Selves J, Pradere B, et al. Octreotide in insulinoma patients: efficacy on hypoglycemia, relationships with Octreoscan scintigraphy and immunostaining with anti-sst2A and antisst5 antibodies. Eur J Endocrinol. 2005;152(5):757-67. https://doi.org/10.153 0/eje.1.01901.

8. Yu L, Zhang J, Guo X, Chen X, He Z, He Q. ARMC5 mutations in familial and sporadic primary bilateral macronodular adrenal hyperplasia. PLoS One. 2018;13(1):e0191602 Published 2018 Jan 25.

9. Zhang Q, Cui L, Gao JP, Yan WH, Jin N, Chen K, et al. Whole-genome sequencing revealed armadillo repeat containing 5 (ARMC5) mutation in a
Chinese family with ACTH-independent macronodular adrenal hyperplasia. Endocr J. 2018;65(3):269-79. https://doi.org/10.1507/endocrj.EJ17-0317.

10. Zhu J, Cui L, Wang W, Hang XY, Xu AX, Yang SX, et al. Whole exome sequencing identifies mutation of EDNRA involved in ACTH-independent macronodular adrenal hyperplasia. Fam Cancer. 2013;12(4):657-67. https:// doi.org/10.1007/s10689-013-9642-y.

11. Newell-Price J, Bertagna X, Grossman AB, Nieman LK. Cushing's syndrome. Lancet. 2006;367(9522):1605-17. https://doi.org/10.1016/S0140-673 6(06)68699-6

12. Gagliardi L, Hotu C, Casey G, Braund WJ, Ling KH, Dodd T, et al. Familial vasopressin-sensitive ACTH-independent macronodular adrenal hyperplasia (VPs-AIMAH): clinical studies of three kindreds. Clin Endocrinol (Oxf). 2009; 70(6):883-91. https://doi.org/10.1111/j.1365-2265.2008.03471.x.

13. Esquela-Kerscher A, Slack FJ. Oncomirs - microRNAs with a role in cancer. Nat Rev Cancer. 2006;6(4):259-69. https://doi.org/10.1038/nrc1840.

14. He L, Hannon GJ. MicroRNAs: small RNAs with a big role in gene regulation [published correction appears in Nat rev genet. 2004 Aug;5(8):631]. Nat Rev Genet. 2004;5(7):522-31. https://doi.org/10.1038/nrg1379.

15. Esteller M. Non-coding RNAs in human disease. Nat Rev Genet. 2011;12(12): 861-74. https://doi.org/10.1038/nrg3074.

16. Bartel DP. MicroRNAs: genomics, biogenesis, mechanism, and function. Cell. 2004;116(2):281-97. https://doi.org/10.1016/S0092-8674(04)00045-5.

17. Rosenfeld N, Aharonov R, Meiri E, Rosenwald S, Spector Y, Zepeniuk M, et al. MicroRNAs accurately identify cancer tissue origin. Nat Biotechnol. 2008; 26(4):462-9. https://doi.org/10.1038/nbt1392.

18. Kent OA, Mendell JT. A small piece in the cancer puzzle: microRNAs as tumor suppressors and oncogenes. Oncogene. 2006;25(46):6188-96. https:// doi.org/10.1038/sj.onc.1209913.

19. Calin GA, Ferracin M, Cimmino A, di Leva G, Shimizu M, Wojcik SE, et al. A MicroRNA signature associated with prognosis and progression in chronic lymphocytic leukemia [published correction appears in N Engl J med. 2006 Aug 3;355(5):533]. N Engl J Med. 2005;353(17):1793-801. https://doi.org/10.1 056/NEJMoa050995.

20. Bloomston M, Frankel WL, Petrocca F, Volinia S, Alder H, Hagan JP, et al. MicroRNA expression patterns to differentiate pancreatic adenocarcinoma from normal pancreas and chronic pancreatitis. JAMA. 2007;297(17):1901-8. https://doi.org/10.1001/jama.297.17.1901.

21. Hu Z, Chen J, Tian T, Zhou X, Gu H, Xu L, et al. Genetic variants of miRNA sequences and non-small cell lung cancer survival. J Clin Invest. 2008;118(7): 2600-8. https://doi.org/10.1172/JCl34934.

22. Danza K, De Summa S, Pinto $R$, et al. TGFbeta and miRNA regulation in familial and sporadic breast cancer. Oncotarget. 2017:8(31):50715-23 Published 2017 Jan 30

23. Mian C, Pennelli G, Fassan M, Balistreri M, Barollo S, Cavedon E, et al. MicroRNA profiles in familial and sporadic medullary thyroid carcinoma: preliminary relationships with RET status and outcome. Thyroid. 2012;22(9): 890-6. https://doi.org/10.1089/thy.2012.0045.

24. Caldas J, Kaski S. Hierarchical generative biclustering for microRNA expression analysis. J Comput Biol. 2011;18(3):251-61. https://doi.org/10.1 089/cmb.2010.0256.

25. Lu J, Getz G, Miska EA, Alvarez-Saavedra E, Lamb J, Peck D, et al. MicroRNA expression profiles classify human cancers. Nature. 2005;435(7043):834-8. https://doi.org/10.1038/nature03702.

26. Miska EA, Alvarez-Saavedra E, Townsend M, Yoshii A, Šestan N, Rakic P, et al. Microarray analysis of microRNA expression in the developing mammalian brain. Genome Biol. 2004;5(9):R68. https://doi.org/10.1186/ gb-2004-5-9-r68.

27. Bimpaki El, lliopoulos D, Moraitis A, Stratakis CA. MicroRNA signature in massive macronodular adrenocortical disease and implications for adrenocortical tumourigenesis. Clin Endocrinol (Oxf). 2010;72(6):744-51. https://doi.org/10.1111/j.1365-2265.2009.03725.x.

28. Almeida MQ, Harran M, Bimpaki El, Hsiao HP, Horvath A, Cheadle C, et al. Integrated genomic analysis of nodular tissue in macronodular adrenocortical hyperplasia: progression of tumorigenesis in a disorder associated with multiple benign lesions. J Clin Endocrinol Metab. 2011;96(4): E728-38. https://doi.org/10.1210/jc.2010-2420.

29. Lacroix A. ACTH-independent macronodular adrenal hyperplasia. Best Pract Res Clin Endocrinol Metab. 2009;23(2):245-59. https://doi.org/10.1016/j. beem.2008.10.011.

30. Mogilyansky E, Rigoutsos I. The miR-17/92 cluster: a comprehensive update on its genomics, genetics, functions and increasingly important and 
numerous roles in health and disease. Cell Death Differ. 2013;20(12):160314. https://doi.org/10.1038/cdd.2013.125.

31. Guo L, Xu J, Qi J, Zhang L, Wang J, Liang J, et al. MicroRNA-17-92a upregulation by estrogen leads to Bim targeting and inhibition of osteoblast apoptosis. J Cell Sci. 2013;126(Pt 4):978-88. https://doi.org/1 $0.1242 /$ jcs. 117515 .

32. Shi C, Qi J, Huang P, Jiang M, Zhou Q, Zhou H, et al. MicroRNA-17/20a inhibits glucocorticoid-induced osteoclast differentiation and function through targeting RANKL expression in osteoblast cells. Bone. 2014;68:6775. https://doi.org/10.1016/j.bone.2014.08.004.

33. Xing W, Hao L, Yang X, Li F, Huo H. Glucocorticoids induce apoptosis by inhibiting microRNA cluster miR-17-92 expression in chondrocytic cells. Mol Med Rep. 2014;10(2):881-6. https://doi.org/10.3892/mmr.2014.2253.

34. Xiao C, Srinivasan L, Calado DP, Patterson HC, Zhang B, Wang J, et al. Lymphoproliferative disease and autoimmunity in mice with increased miR17-92 expression in lymphocytes. Nat Immunol. 2008;9(4):405-14. https:// doi.org/10.1038/ni1575.

35. Harada M, Pokrovskaja-Tamm K, Söderhäll S, Heyman M, Grander D, Corcoran M. Involvement of miR17 pathway in glucocorticoid-induced cell death in pediatric acute lymphoblastic leukemia. Leuk Lymphoma. 2012; 53(10):2041-50. https://doi.org/10.3109/10428194.2012.678004.

36. Smith LK, Shah RR, Cidlowski JA. Glucocorticoids modulate microRNA expression and processing during lymphocyte apoptosis. J Biol Chem. 2010; 285(47):36698-708. https://doi.org/10.1074/jbc.M110.162123.

37. Tessel MA, Benham AL, Krett NL, Rosen ST, Gunaratne PH. Role for microRNAs in regulating glucocorticoid response and resistance in multiple myeloma. Horm Cancer. 2011;2(3):182-9. https://doi.org/10.1007/s12672011-0072-8.

38. Faustino RS, Cheung P, Richard MN, Dibrov E, Kneesch AL, Deniset JF, et al. Ceramide regulation of nuclear protein import. J Lipid Res. 2008;49(3):65462. https://doi.org/10.1194/jr.M700464-JLR200.

39. Wood PA, Yang X, Hrushesky WJ. Clock genes and cancer. Integr Cancer Ther. 2009;8(4):303-8. https://doi.org/10.1177/1534735409355292.

40. Krugluger W, Brandstaetter A, Kállay E, Schueller J, Krexner E, Kriwanek S, et al. Regulation of genes of the circadian clock in human colon cancer: reduced period-1 and dihydropyrimidine dehydrogenase transcription correlates in high-grade tumors. Cancer Res. 2007;67(16):7917-22. https:// doi.org/10.1158/0008-5472.CAN-07-0133.

41. Charmandari E, Chrousos GP, Lambrou Gl, Pavlaki A, Koide H, Ng SSM, et al. Peripheral CLOCK regulates target-tissue glucocorticoid receptor transcriptional activity in a circadian fashion in man. PLoS One. 2011;6(9): e25612. https://doi.org/10.1371/journal.pone.0025612.

42. Kino T, Chrousos GP. Acetylation-mediated epigenetic regulation of glucocorticoid receptor activity: circadian rhythm-associated alterations of glucocorticoid actions in target tissues. Mol Cell Endocrinol. 2011;336(1-2): 23-30. https://doi.org/10.1016/j.mce.2010.12.001.

43. Nader N, Chrousos GP, Kino T. Circadian rhythm transcription factor CLOCK regulates the transcriptional activity of the glucocorticoid receptor by acetylating its hinge region lysine cluster: potential physiological implications. FASEB J. 2009;23(5):1572-83. https://doi.org/10.1096/fj.08-11 7697.

\section{Publisher's Note}

Springer Nature remains neutral with regard to jurisdictional claims in published maps and institutional affiliations.

\section{Ready to submit your research? Choose BMC and benefit from:}

- fast, convenient online submission

- thorough peer review by experienced researchers in your field

- rapid publication on acceptance

- support for research data, including large and complex data types

- gold Open Access which fosters wider collaboration and increased citations

- maximum visibility for your research: over $100 \mathrm{M}$ website views per year

At BMC, research is always in progress.

Learn more biomedcentral.com/submissions 